\title{
BMJ Open The effect of smell and taste of milk during tube feeding of preterm infants (the Taste trial): a protocol for a randomised controlled trial
}

\author{
Friederike Beker, ${ }^{\oplus 1,2}$ Judith Macey, ${ }^{\oplus 1}$ Helen Liley, ${ }^{\oplus, 2}$ Ian Hughes, ${ }^{3}$ \\ Peter G Davis, ${ }^{4,5}$ Emily Twitchell, ${ }^{5}$ Susan Jacobs ${ }^{4,5}$
}

To cite: Beker F, Macey J, Liley $\mathrm{H}$, et al. The effect of smell and taste of milk during tube feeding of preterm infants (the Taste trial): a protocol for a randomised controlled trial. BMJ Open 2019;9:e027805. doi:10.1136/ bmjopen-2018-027805

- Prepublication history and additional material for this paper are available online. To view please visit the journal (http:// dx.doi.org/10.1136/bmjopen2018-027805).

Received 12 November 2018 Revised 12 June 2019 Accepted 13 June 2019

Check for updates

(c) Author(s) (or their employer(s)) 2019. Re-use permitted under CC BY-NC. No commercial re-use. See rights and permissions. Published by BMJ.

${ }^{1}$ Mater Research Institute, University of Queensland, South Brisbane, Queensland, Australia ${ }^{2}$ Neonatal Critical Care Unit, Mater Mothers' Hospital, Brisbane, Queensland, Australia ${ }^{3}$ Office for Research Governance and Development, Gold Coast University Hospital, Southport, Queensland, Australia

${ }^{4}$ Neonatal Intensive Care Unit and Newborn Research, The Royal Women's Hospital, Melbourne, Victoria, Australia ${ }^{5}$ Clinical Sciences Research, Murdoch Childrens Research Institute, Parkville, Victoria, Australia

Correspondence to Dr Friederike Beker; friederike.beker@mater.org.au

\section{ABSTRACT}

Introduction Smell and taste of milk are not generally considered when tube feeding preterm infants. Preterm infants have rapid growth, particularly of the brain, and high caloric needs. Enteral feeding is often poorly tolerated which may lead to growth failure and longterm neurodevelopmental impairment. Smell and taste are strong stimulators of digestion and metabolism. We hypothesise that regular smell and taste during tube feeding will improve weight z-scores of very preterm infants at discharge from hospital.

Methods and analysis Taste is a randomised, unblinded two-centre trial. Infants born at $<29$ weeks' gestation and/or $<1250 \mathrm{~g}$ at birth and admitted to a participating neonatal intensive care unit are eligible. Randomisation occurs before infants receive two hourly feeds for 24 hours. Infants are randomised to either smell and taste of milk with each tube feed or tube feeding without the provision of smell and taste. The primary outcome is weight z-score at discharge. Secondary outcomes include: days to full enteral feeds, duration of parenteral nutrition, rate of late-onset sepsis, post menstrual age at removal of nasogastric tube and at discharge from hospital, anthropometric data and neurodevelopmental outcomes at 2 years of corrected age.

Ethics and dissemination Human Research Ethics Committees of Mater Misericordiae (trial reference number: HREC/16/MHS/112) and the Royal Women's Hospital (trial reference number: 17/21) last approved the trial protocol (version 4.2; Date: 18 December 2018) and recruitment commenced in May 2017 and November 2017 , respectively. The trial results will be published in a peer-reviewed journal and will be presented at national and international conferences.

Trial registration number ACTRN12617000583347.

\section{INTRODUCTION}

\section{Background and rationale}

Close to 7000 infants are born before term and admitted to neonatal intensive care units (NICUs) across Australia and New Zealand each year. ${ }^{1}$ Preterm infants have an impaired ability to breathe, suck and swallow in a coordinated fashion. This immaturity presents a
Strength and limitations of this study

- This trial is the first adequately powered randomised trial to investigate the effects of smell and taste during tube feeding of very preterm infants.

- Blinding of the allocated intervention is not feasible; therefore, an objective criterion for the primary outcome (weight z-score at discharge) was specified.

- Effects of other smell or taste experiences, for example, encountered during skin-to-skin time, with reflux or due to the tastes of varying milk types are not considered and occur in both study groups.

- Smelling and tasting of milk is an uncomplicated and potentially cost-effective intervention that may have a number of beneficial health effects such as improved weight gain and feed tolerance as well as a reduction in length of hospital stay.

significant challenge to the provision of effective nutrition, commonly leading to postnatal growth failure. ${ }^{2}$ Brain growth in the last 4 to 8 weeks of gestation is extremely rapid and is crucial for later development. Malnutrition during a vulnerable phase of brain development leads to a reduced number of neurons and later behaviour, learning and memory problems. In preterm infants, this extremely rapid phase of brain growth occurs ex utero at a time when providing adequate nutrition is challenging. Thus, optimising postnatal growth may result in improved neurodevelopmental outcomes. ${ }^{34}$

\section{Taste and smell in the NICU}

Pleasant odours and tastes, such as those encountered with a good meal or a familiar person, have an enormous influence on our own daily well-being. Despite our own understanding of the role of taste and smell in our lives, preterm infants are usually only exposed to the smell of the mother's skin or breast milk once the infant is well enough to be removed from the incubator for skin-to-skin care and 
breast feeding. The frequency and duration of such skin-to-skin care is dependent on the philosophy of the NICU and individual staff. ${ }^{5}$ The infant's most common smell experience is often restricted to the odours of the direct environment, commonly excrement and antiseptics. Taste experiences may be dominated by rubber and plastic from feeding and breathing tubes in the mouth or associated with discomfort and pain, when breastmilk or sucrose is given for pain relief. ${ }^{67}$ Thus, preterm infants are not only deprived of the pleasures of smell and taste but it is also possible that placement of milk feeds directly into the stomach via a feeding tube without any food anticipation may impact on metabolism and early nutritional learning.

\section{Food anticipation and the cephalic phase response}

Anticipation of food activates the digestive system. Pavlov famously explored and described this phenomenon over a hundred years ago. ${ }^{8}$ Sham feeding, the mere taste of food in the oral cavity of dogs, led to the production of gastric secretions. Pavlov also confirmed that the sight of food or even unrelated signals such as the sound of a bell could elicit the same strong response, as long as the dog was conditioned and knew that the bell was related to food intake. In an experiment analogous to the gastric feeding of preterm infants, Pavlov also placed bread into the stomach of his dogs through a tube, without the dog being aware. The bread remained undigested in the dogs' stomachs for up to 1 hour. Furthermore, Pavlov stated that a food ingested by a dog only acted as a stimulus when it 'suited the dog's taste'. He concluded that the response of the stomach to the anticipation of food depended on the presence of appetite. ${ }^{89}$ This activation of the digestive system by anticipation of food has been named the 'cephalic response'. ${ }^{11}$ Decades of ongoing research have revealed that the cephalic response plays an even more complex role in nutrition, from early nutritional learning to improved nutrient absorption, increased stomach and gut motility, anticipatory secretion of insulin with tighter blood glucose control and the release of appetite, digestive and metabolic hormones such as leptin, ghrelin, insulin and gastrin. ${ }^{12} 13$

\section{Smell and taste in preterm infants}

Preterm infants are believed to have flavour perception. Functional taste receptors are present from 18 weeks' postmenstrual age (PMA) and flavour perception is established around 24 weeks' PMA. Changes in tissue oxygenation by near-infrared spectroscopy have been detected in term and preterm infants $>32$ weeks' PMA in response to odours, with different responses occurring to odours rated as pleasant or unpleasant. ${ }^{145}$

Amniotic fluid and breast milk have flavours that reflect the foods, spices and beverages consumed by the mother. ${ }^{16}$ Infants exposed to those flavours during late pregnancy and early infancy exhibit food preference to such flavours, some persisting into adulthood. ${ }^{18}$ It is well known that toddlers prefer the flavour composition they have been exposed to in utero and during breast feeding (the mother's diet) as infants. ${ }^{19}$ Similarly, exposure to alcohol in late pregnancy makes alcohol more palatable in later life and even increases intake. ${ }^{20}{ }^{21} \mathrm{It}$ is also suspected that intrauterine and postnatal exposure to fructose increases the rate of obesity later by altering feeding behaviour and appetite control as well as neuroendocrine function. ${ }^{22}$

Early priming of the olfactory and gustatory systems is critical, as the ability to 'taste' chemical compounds guides the amount of food eaten and is imperative for the evaluation of food quality. Once food intake is expected or commenced, the brainstem and higher centres activate the cephalic phase response and release appetite hormones in saliva. ${ }^{23}$ These salivary hormones are postulated to play a role in metabolism; indeed, impaired oral nutrient sensing is associated with increased energy intake and a greater body mass index. ${ }^{24}$

Preterm infants do not have the opportunity to experience the most basic of stimuli and sensation associated with feeding: hunger, satiety, taste and smell. NICU clinicians regulate feed times, frequency and volumes of feed. Milk is delivered through a gastric tube until infants are mature enough to attempt breastfeeding bypassing the gustatory and olfactory receptors that are involved in stimulating many of the preprandial responses outlined above.

\section{Relevant clinical trials in the NICU environment}

There are three prior studies in preterm infants in which taste may have been a variable affecting time to full enteral feeds. Rodriguez et al reported as a secondary outcome in a randomised study of 16 extremely low birthweight (ELBW; $<1000 \mathrm{~g}$ ) infants that infants receiving two-hourly oropharyngeal colostrum for 48 hours in the first days after birth reached full enteral feeds significantly faster than the control group, receiving water instead of colostrum. ${ }^{25}$ However, another similar study of 48 ELBW infants who received three-hourly oropharyngeal colostrum for 3 days in the first days after birth did not find any difference in time to full enteral feeds between the intervention and control groups. ${ }^{26}$ Both studies provided oropharyngeal colostrum for only a few days with the primary outcome being immunological effects. Neither of the studies reported if the colostrum was given in combination with tube feeds.

Another study investigated the effect of 14 days of a sweetened pacifier versus a plain pacifier on weight gain in infants born less than 34 weeks' PMA with a birth weight of more than $1250 \mathrm{~g} .{ }^{27}$ There were no statistically significant differences between groups.

A pilot trial, preceding this study, demonstrated that smell and taste with every tube feed reduced the time to full enteral feeds in very low birthweight (VLBW, $<1500 \mathrm{~g}$ ) infants. Infants who had been exposed to regular smell and taste of their milk feed also had higher weight z-scores at discharge, a crucial outcome, as higher weight z-scores 
are associated with better long-term neurodevelopmental outcomes. ${ }^{3} 28$

Following this pilot study, Bloomfield et al included 'smell and taste with tube feeding' into their study protocol examining early feeding practices in moderate to late preterm infants and their effect on nutritional, metabolic and neurodevelopmental outcomes. ${ }^{29}$ The same group also recently published a Cochrane Database of Systematic Reviews protocol to examine the effects of smell and taste with tube feeding. ${ }^{30}$

\section{Summary and rationale}

Few NICUs routinely provide infants with the smell and/or taste of their milk with tube feeds, despite the assumption that premature infants can taste and smell, and despite our own regular indulgence in smell and taste perception. It is common for smell and/or taste to be provided on an ad hoc basis, for pain relief, mouth care and/or occasionally with tube feeding. Smell and taste strongly elicit the cephalic phase response and may have the potential to improve milk tolerance, digestion and metabolism in VLBW infants. The few published studies that investigated the effects of taste on tube feeding expose infants only for a few days, include only late preterm infants or have small sample sizes. This trial is powered to demonstrate the effects of smell and taste of milk with every tube feed on the weight z-score at discharge. Other important outcomes include: length of stay in hospital, duration of parenteral nutrition and late-onset sepsis.

\section{METHODS AND ANALYSIS}

Trial design and setting

The Taste trial is a two-centre, parallel-group randomised superiority trial, including preterm infants admitted to the NICU at the Mater Mothers' Hospital (MMH) in Brisbane and the NICU at the Royal Women's Hospital (RWH) in Melbourne, both in Australia. The Taste trial is registered with the Australian and New Zealand Clinical Trials Registry (ACTRN 12617000583347: online supplementary file 1 ).

\section{Participant and public involvement}

Feedback and discussions from families enrolled in the pilot study aided in the development of the research question and outcome measures as well as in assessing the burden of the intervention. Governance regulations did not allow for the involvement of relevant patient families in the planning of or in the recruitment to this study. Results of this study will be published on the MMH and RWH website and social media.

\section{Eligibility criteria}

Inclusion criteria

Male and female preterm infants born at less than 29 weeks' PMA and/or birth weight less than $1250 \mathrm{~g}$ with written informed parental/guardian consent. Consent must be obtained before two-hourly or more frequent feeds have been initiated for 24 hours.

\section{Exclusion criteria}

Congenital conditions associated with the digestive system requiring surgery shortly after birth, for example, gastroschisis, any malformation requiring a stoma at birth (eg, anal atresia), oesophageal atresia; congenital conditions leading to impaired growth, for example, trisomy 21 , trisomy 18 or salt wasting enteropathy.

\section{Interventions}

\section{Treatment group}

Infants in the treatment group receive smell and taste with every tube feed by the bedside nurse:

- Smell: a drop of milk on a gauze swab will be placed as close as possible to the infant's nose, without touching. The intervention will be ceased at 32 weeks' PMA in order to comply with safe sleeping recommendations. ${ }^{31}$

- Taste: a cotton wool bud soaked in milk will be placed on the infant's tongue if the infant is less than 32 weeks' PMA. From 32 weeks' PMA, $0.2 \mathrm{~mL}$ of milk will be given to the infant directly on the tongue.

If the infant is asleep, the smell is given as described above. For the taste, the milk is held onto the infants' lips. If the infant shows any interest, the milk (cotton bud or syringe) is placed in the infants' mouth.

\section{Control group}

Infants receive routine care and do not have any milk in the mouth with tube feeding. Milk for tasting with nasogastric tube feeds can only be given if prescribed by a speech pathologist, usually not before 38 weeks' PMA. The control group resembles routine care and was therefore chosen as comparator.

All infants, treatment and control group, are allowed to suck feed, receive sucrose, have skin-to-skin care and other contact with their parents, smell blankets provided by parents and/or suck on pacifiers at any time if parental consent is provided. The trial ends with the removal of the nasogastric tube. Parents can decide if they want to give oral milk with tube feeds or not if an infant is discharged home on nasogastric tube feeds.

Withdrawal of consent leads to routine care currently practiced in the NICUs, that is, nasogastric tube feeding without the provision of smell and taste of milk. Therefore, withdrawal of consent only in the treatment group leads to a change in the infant's care.

Bedside instructions for both allocation groups are placed at the cot side to ensure adherence to the trial protocol. Research nurses check regularly that instructions remain at the bedside and that nurses adhere to those instructions. Site investigators ensure adherence to the trial protocol. Despite regular follow-up and endorsement of the trial protocol by nursing staff, treatment change may occur but will not lead to trial exclusion. 


\section{Primary outcome}

- Weight z-score at discharge from hospital.

\section{Secondary outcomes}

- Time (days) to full enteral feeds $(120 \mathrm{~mL} / \mathrm{kg} / \mathrm{d}$ for at least 24 hours)

- Duration of parenteral nutrition (days) total, and first episode

- Rate of late-onset sepsis, diagnosed after 24hours of life in a symptomatic infant with positive blood culture, cerebrospinal fluid or sterile collected urine, treated for a minimum of 5 days with antibiotics. Potential contaminants (eg, coagulase - negative staphylococci) will be included if the infant in addition has a neutrophil left shift of $\geq 20 \%$ and/or C-reactive protein is $\geq 10 \mathrm{mg} / \mathrm{L}$.

- Cumulative duration of antibiotic therapy (days)

- PMA at removal of nasogastric tube

- PMA at discharge home from hospital

- Type of feeding at different time points (eg, type of milk given)

- Rate and severity of retinopathy of prematurity

- Rate and severity of necrotising enterocolitis

- Rate and severity of intraventricular haemorrhage

- Rate of chronic lung disease at 36 weeks PMA

- Spontaneous intestinal perforation

- Rate of treated patent ductus arteriosus

- Anthropometric data at different time points

- Weight at 28 days and 36 weeks' PMA

- Head circumference at 36 weeks' PMA and at discharge home

- Length at 36 weeks' PMA and at discharge home
- Respiratory support in hours (continuous positive airway pressure or high flow nasal cannula, and endotracheal respiratory support)

- Data will be collected from infants assessed in the long-term follow-up programme at 1 and 2 years corrected age (CA) (eg, anthropometric data, respiratory support, type of feeding, cerebral palsy, level within the Gross Motor Function Classification System, hearing and vision assessments, Bayley III results)

- Rate of breast feeding at 3,6 and 12 months of CA.

\section{Participant timeline}

The schedule of enrolment, interventions and assessments is presented in table 1 .

\section{Sample size calculation}

Sample size calculation for the Taste trial is based on detecting an improvement in weight z-score at discharge from a mean of -0.31 to -0.1 with a type I error of $5 \%$ and $90 \%$ power. Multiples are randomised together to the same study group. Therefore, twins and triplets were considered in the sample size calculation with a mother's infants from one pregnancy defined as a cluster. The pilot study had a mean cluster size of 1.08 with an intracluster correlation coefficient of 0.35 and an SD in weight z-score at discharge of 0.58 . Given these criteria, based on a two-sided generalised estimating equations (GEE) model, the required sample size was calculated to be 165 for each group. ${ }^{32}$ This sample size is inflated and controls for clustering within multiple births.

Table 1 Schedule of enrolment, interventions and assessments

\begin{tabular}{|c|c|c|c|c|c|c|c|c|c|}
\hline \multirow[b]{3}{*}{ Time point } & \multicolumn{9}{|l|}{ Trial period } \\
\hline & \multirow{2}{*}{$\begin{array}{l}\text { Enrolment } \\
-t_{1}\end{array}$} & \multirow{2}{*}{$\begin{array}{l}\text { Allocation } \\
t_{0}\end{array}$} & \multicolumn{6}{|c|}{ Post allocation } & \multirow{2}{*}{$\frac{\text { Closeout }}{t_{x}}$} \\
\hline & & & $t_{1}$ & $t_{3}$ & $t_{4}$ & $t_{5}$ & $t_{6}$ & $t_{7}$ & \\
\hline Eligibility screen & $x$ & & & & & & & & \\
\hline Informed consent & $x$ & & & & & & & & \\
\hline \multicolumn{10}{|l|}{ Interventions } \\
\hline \multicolumn{10}{|l|}{$\begin{array}{l}\text { Smell and taste with } \\
\text { tube feeding }\end{array}$} \\
\hline \multicolumn{10}{|l|}{ Routine care } \\
\hline Secondary outcomes & & & $x$ & $x$ & $x$ & $x$ & $x$ & $x$ & $x$ \\
\hline
\end{tabular}

t1: before allocation; t0: time of allocation/randomisation; t1: time of full enteral feeds; t2: anthropometric data at 36 weeks' PMA; 3 : time of discharge; t4: 3 months CA; t5: 6 months CA; t6: 1 year CA; t7: 2 years CA; tx: 2 years CA for infants eligible for the long-term follow-up programme, 1 year for infants not eligible for the follow-up programme but parents consented to be contacted for breast feeding rates, time of discharge home from hospital for all other infants. 


\section{Recruitment}

The study team member will identify potential participants for the trial and approach their parents for written consent. Parents will not be approached for consent antenatally, but they may be informed about the trial. However, if parents indicate that they do want their infant to participate in the trial, a participant information and consent form (PICF-online supplementary file 2) will be provided.

Participants will be actively recruited after birth and parents approached for written consent by a study team member. Potential participants for the trial will be identified from the inpatient list of the NICUs on a daily basis. Parents will be approached when they have recovered from the stress of birth and when they are able to consent. Parents will have the ability to consider participation, discuss the trial with their friends, family and local general practitioner, ask questions and decide to consent or not to the trial without any consequences to the care of their infant. Clinical care of the infant will always take priority over any research study and wherever possible, consent will be obtained by a member of the study team not directly involved in the infant's clinical care.

\section{Randomisation}

A randomisation sequence of treatment or control with variable block sizes ${ }^{2-6}$ was generated by $\mathrm{IH}$ using the ralloc command of Stata V.14 (College Station, Texas, USA). Randomisation is stratified by site, sex and PMA $(<27$ weeks' PMA and >/=27 weeks' PMA). Each participating centre is provided with sequentially numbered, sealed, opaque, envelopes containing the assigned treatment allocation. The envelope is opened after parental consent has been given, immediately before the trial commences. One envelope is opened for each set of multiple births.

\section{Blinding}

Treatment allocation and the primary outcome are not blinded in this trial. Blinding of the treatment allocation was considered but it was concluded that it was not feasible for the intervention tested. A robust primary outcome was chosen with the aim to prevent observer bias while it is acknowledged that the potential for "treatment leakage' still exists. To mitigate this concern, we will ensure that clinical care teams, researchers and parents/ caregivers are provided education regarding the importance of maintaining the integrity of the randomisation of the trial.

The following secondary outcomes are assessed by clinicians blinded to the infant's allocated group: retinopathy of prematurity, x-ray findings required to determine the severity of necrotising enterocolitis, intraventricular haemorrhage, presence of chronic lung disease, spontaneous intestinal perforation, respiratory support in hours, outcomes from long-term follow-up programme from eligible infants at 1 and 2 years CA.

\section{Data management}

Data will be sourced from each participant's observation chart, clinical care team notes, medical records and verbally from parents. Each infant will be assigned a study number and data will be collected under that study number. Data will be deidentified when entered onto a paper case record form, then transferred by the data manager to an excel spread sheet and stored on a password-protected computer on the MMH computer network. Each data set will be checked by the principal investigator for plausibility and data range checks are applied in the database as appropriate.

The MMH Human Research Ethics Committee reviewed the protocol and the pilot study and advised that a data monitoring committee, an interim analysis and stopping guidelines were not required for this trial.

\section{Statistical methods}

Statistical analysis will be performed by the authors Hughes and Beker with assistance of other study group members. Data will be exported from an excel spreadsheet to a statistical package for analysis (Stata; College Station, Texas, USA). Data will be analysed on an intention-to-treat basis. All randomised infants will be included in the primary analysis, unless consent has been withdrawn. Data of deceased infants will be included in the analysis if the respective outcome is achieved.

Univariate and multivariable GEE analyses will be used for the primary outcome, weight z-scores at discharge from hospital and other continuous secondary outcome measures. Time to full enteral feeds will be analysed using a multilevel survival analysis (mestreg command in Stata) ${ }^{28}$ Secondary outcomes with categorical data will be analysed using a mixed effects logistic regression (melogit Stata command). Subgroup analysis will be performed based on sex and PMA for the primary outcome and selected secondary outcomes.

All outcomes will be assessed against a hypothesis of superiority.

\section{Harms}

Principal investigators/their delegates will be responsible for all safety reporting. Study infants are at high risk and rely on intensive care of their medical problems. Deaths of study infants will be reported to the approving HREC and governance department within 24 hours of knowing by the site principal investigator/delegate. This includes adjudication of the likelihood of the event being related to the involvement in this trial.

In both participating NICUs, clinical incidents are reviewed by the Patient Safety Units. Trends and concerns regarding patient safety are analysed and the results shared with the NICUs to prevent patient harm. Principal investigators will be informed by the Patient Safety Units should there be any concern in regards to the safety of the Taste trial. The Taste trial has no established external study monitoring committee. 


\section{DISCUSSION}

Exposure of preterm infants to the smell and taste of milk is infrequently considered by clinicians or researchers. Smell and taste of food prepares the body for food intake, digestion and metabolism and may improve important clinical outcomes of preterm infants who are challenged by suboptimal weight gain and poor enteral milk tolerance. ${ }^{28}$

Taste is the first adequately powered trial to test the effect of smell and taste in very preterm infants. Use of a placebo in the control group has proven difficult. Pavlov's experiments with dogs have demonstrated that multiple sensory inputs, not related to food intake, can elicit a cephalic phase response. The offer of normal saline or water taste on a cotton bud or via syringe is therefore not appropriate for the control group. Due to the lack of blinding, a robust primary outcome (weight z-scores at discharge from hospital) was selected.

It is widely accepted that weight gain is based on a balance of calories provided paired with the metabolic needs of the infants. Regular smell and taste with feeding may play an additional role in nutrition by modifying digestion and absorption as well as influence early nutritional learning in the form of appetite and satiety regulation. The selection of weight z-scores at discharge as the primary outcome measure for this study is based on that assumption supported by the results of our pilot trial. ${ }^{28}$ Weight at discharge also seemed more relevant than short-term nutritional outcomes as a relationship between weight at discharge and long-term neurodevelopmental outcomes has been reported. ${ }^{23}$

Full enteral feeds are reported as secondary outcome at $120 \mathrm{~mL} / \mathrm{kg} / \mathrm{d}$ instead of 150 or $160 \mathrm{~mL} / \mathrm{kg} / \mathrm{d}$ as recommended by the COMMENT (Consensus Group on Outcome Measures Made in Paediatric Enteral Nutrition Clinical Trials) group. Their core data set was developed based on a previous proposal by the European Society for Paediatric Gastroenterology, Hepatology and Nutrition (ESPGHAN) Committee on Nutrition. ${ }^{33}$ A rationale for choosing $120 \mathrm{~mL} / \mathrm{kg} / \mathrm{d}$ over higher amounts of milk may be the fluid restriction of infants for non-nutritionrelated reasons to below $150 \mathrm{~mL} / \mathrm{kg} / \mathrm{d}$ (less likely below $120 \mathrm{~mL} / \mathrm{kg} / \mathrm{d}$ ) so the time to reach $150 \mathrm{~mL} / \mathrm{kg} / \mathrm{d}$ may be delayed until fluid restriction is ceased.

If smell and taste with tube feeding are shown to be beneficial for very preterm infants, this straight forward intervention may easily be adopted by NICUs and not only improve clinical outcomes, but also save costs and resources.

\section{ETHICS AND DISSEMINATION \\ Research ethics}

The HRECs of MML and RWH approved of the study protocol (version 3, 8 May 2017), trial reference number HREC/16/MHS/112 and trial reference number 17/21, respectively. Both hospitals also granted governance approval.

\section{Dissemination of results}

The results of the trial will be published in a peer-reviewed journal and will be presented at national and international conferences. Authorship will be determined in line with the International Committee of Medical Journal Editors guidelines. A data sharing agreement will be in place to allow all study group members to access the final trial data set. Access to the participant-level data set may be granted if an appropriate data sharing agreement is arranged.

Acknowledgements We thank the families for their encouragement and participation in the pilot trial and nurses and medical staff for their ambition to include the study into their daily care routine.

Contributors FB conceived of the study. FB, IH and SEJ developed the study design and FB, HL, PGD, JM, ET and SJ facilitated study implementation. IH provided statistical expertise in clinical trial design and together with FB planned the statistical analysis. All authors contributed to refinement of the study protocol and approved the final manuscript.

Funding The primary trial sponsor is Mater Misericordiae, contact: CEO Mater Research, Governance 0ffice, email: research.governance@mater.uq.edu.au, phone: +61731633769 . This work is supported by Mater Research Institute, the Department of Newborn Research at the RWH and the National Health and Medical Research Council (NHMRC) Program Grant (\#1113902). PGD is supported by an NHMRC Practitioner Fellowship. The Royal Australasian College of Physicians and Paediatricians - Queensland Branch provides funding for statistical support.

Competing interests PGD received salary support from Australia's national research funding agency.

Patient consent for publication Not required.

Provenance and peer review Not commissioned; externally peer reviewed.

Open access This is an open access article distributed in accordance with the Creative Commons Attribution Non Commercial (CC BY-NC 4.0) license, which permits others to distribute, remix, adapt, build upon this work non-commercially, and license their derivative works on different terms, provided the original work is properly cited, appropriate credit is given, any changes made indicated, and the use is non-commercial. See: http://creativecommons.org/licenses/by-nc/4.0/.

\section{REFERENCES}

1. Chow SS, Creighton P, Kander V, et al. 2016 Report of the Australian and New Zealand Neonatal Network. 2018. http://www.anznn.net

2. Harding JE, Derraik JG, Berry MJ, et al. Optimum feeding and growth in preterm neonates. J Dev Orig Health Dis 2013;4:215-22.

3. Ehrenkranz RA, Dusick AM, Vohr BR, et al. Growth in the neonatal intensive care unit influences neurodevelopmental and growth outcomes of extremely low birth weight infants. Pediatrics 2006;117:1253-61.

4. Belfort MB, Rifas-Shiman SL, Sullivan T, et al. Infant growth before and after term: effects on neurodevelopment in preterm infants. Pediatrics 2011;128:e899-e906.

5. Hendricks-Muñoz KD, Li Y, Kim YS, et al. Maternal and neonatal nurse perceived value of kangaroo mother care and maternal care partnership in the neonatal intensive care unit. Am J Perinatol 2013;30:875-80.

6. Collados-Gómez L, Ferrera-Camacho P, Fernandez-Serrano E, et al. Randomised crossover trial showed that using breast milk or sucrose provided the same analgesic effect in preterm infants of at least 28 weeks. Acta Paediatr 2018;107:436-41.

7. Stevens B, Yamada J, Lee GY, et al. Sucrose for analgesia in newborn infants undergoing painful procedures. Cochrane Database Syst Rev 2013;1:CD001069.

8. Pavlov I. Ivan Pavlov: Nobel Lecture: Physiology of Digestion. Nobel Media AG. 2014. http://www.nobelprize.org/nobel_prizes/medicine/ laureates/1904/pavlov-lecture.html

9. Bayliss WM. The physiological work of ivan petrovich Pavlov: Abstract of a Lecture given at University College, London. Br Med J 1916;2:799-800.

10. Katschinski M, Dahmen G, Reinshagen M, et al. Cephalic stimulation of gastrointestinal secretory and motor responses in humans. Gastroenterology 1992;103:383-91. 
11. Bruce DG, Storlien LH, Furler SM, et al. Cephalic phase metabolic responses in normal weight adults. Metabolism 1987;36:721-5.

12. Power ML, Schulkin J. Anticipatory physiological regulation in feeding biology: cephalic phase responses. Appetite 2008;50(23):194-206.

13. Teff KL. How neural mediation of anticipatory and compensatory insulin release helps us tolerate food. Physiol Behav 2011;103:44-50.

14. Bartocci M, Winberg J, Ruggiero C, et al. Activation of olfactory cortex in newborn infants after odor stimulation: a functional nearinfrared spectroscopy study. Pediatr Res 2000;48:18-23.

15. Bartocci M, Winberg J, Papendieck G, et al. Cerebral hemodynamic response to unpleasant odors in the preterm newborn measured by near-infrared spectroscopy. Pediatr Res 2001;50:324-30.

16. Varendi H, Porter RH, Winberg J. Natural odour preferences of newborn infants change over time. Acta Paediatr 1997;86:985-90.

17. Mennella JA. Ontogeny of taste preferences: basic biology and implications for health. Am J Clin Nutr 2014;99:704S-11.

18. Haller R, Rummel C, Henneberg S, et al. The influence of early experience with vanillin on food preference later in life. Chem Senses 1999;24:465-7.

19. Mennella JA, Jagnow CP, Beauchamp GK. Prenatal and postnatal flavor learning by human infants. Pediatrics 2001;107:E88

20. Pautassi RM, Nizhnikov ME, Spear NE, et al. Prenatal ethanol exposure leads to greater ethanol-induced appetitive reinforcement. Alcohol 2012;46:585-93.

21. Youngentob SL, Glendinning Jl. Fetal ethanol exposure increases ethanol intake by making it smell and taste better. Proc Natl Acad Sci U S A 2009;106:5359-64

22. Goran MI, Dumke K, Bouret SG, Dumk S, Bouret B, et al. The obesogenic effect of high fructose exposure during early development. Nat Rev Endocrinol 2013;9:494-500.

23. Zolotukhin S. Metabolic hormones in saliva: origins and functions. Oral Dis 2013;19:219-29.
24. Hurtado MD, Sergeyev VG, Acosta A, et al. Salivary peptide tyrosinetyrosine 3-36 modulates ingestive behavior without inducing taste aversion. J Neurosci 2013;33:18368-80.

25. Rodriguez NA, Groer MW, Zeller JM, et al. A randomized controlled trial of the oropharyngeal administration of mother's colostrum to extremely low birth weight infants in the first days of life. Adv Neonatal Care 2011;24:31-5.

26. Lee J, Kim HS, Jung YH, et al. Oropharyngeal colostrum administration in extremely premature infants: an RCT. Pediatrics 2015;135:e357-e366.

27. Mattes RD, Maone T, Wager-Page S, et al. Effects of sweet taste stimulation on growth and sucking in preterm infants. J Obstet Gynecol Neonatal Nurs 1996;25:407-14.

28. Beker F, Opie G, Noble E, et al. Smell and Taste to Improve Nutrition in Very Preterm Infants: A Randomized Controlled Pilot Trial. Neonatology 2017;111:260-6.

29. Bloomfield FH, Harding JE, Meyer MP, et al. The DIAMOND trial - DIfferent Approaches to MOderate \& late preterm Nutrition: Determinants of feed tolerance, body composition and development: protocol of a randomised trial. BMC Pediatr 2018;18:220.

30. Muelbert M, Harding JE, Bloomfield FH. Exposure to the smell and taste of milk to accelerate feeding in preterm infants. Cochrane Database Syst Rev 2018:CD013038.

31. Moon RY. Task Force on Sudden Infant Death Syndrome. SIDS and Other Sleep-Related Infant Deaths: Evidence Base for 2016 Updated Recommendations for a Safe Infant Sleeping Environment. Pediatrics 2016;138:e20162940.

32. Rutterford C, Copas A, Eldridge S. Methods for sample size determination in cluster randomized trials. Int $\mathrm{J}$ Epidemiol 2015;44:1051-67.

33. Koletzko B, Fewtrell M, Gibson R, et al. Core data necessary for reporting clinical trials on nutrition in infancy. Ann Nutr Metab 2015;66:31-5. 